\title{
ANALOG BEAMFORMING WITH ANTENNA SELECTION FOR LARGE-SCALE ANTENNA ARRAYS
}

\author{
Aakash Arora, Christos G. Tsinos, Bhavani Shankar Mysore R, Symeon Chatzinotas and Björn Ottersten \\ Interdisciplinary Centre for Security, Reliability and Trust (SnT), University of Luxembourg \\ email address: \{aakash.arora, christos.tsinos, bhavani.shankar, symeon.chatzinotas, bjorn.ottersten\}@uni.lu
}

\begin{abstract}
In large-scale antenna array (LSAA) wireless communication systems employing analog beamforming architectures, the placement or selection of a subset of antennas can significantly reduce the power consumption and hardware complexity. In this work, we propose a joint design of analog beamforming with antenna selection (AS) or antenna placement (AP) for an analog beamforming system. We approach this problem from a beampattern matching perspective and formulate a sparse unit-modulus least-squares (SULS) problem, which is a nonconvex problem due to the unit-modulus and the sparsity constraints. To that end, we propose an efficient and scalable algorithm based on the majorization-minimization (MM) framework for solving the SULS problem. We show that the sequence of iterates generated by the algorithm converges to a stationary point of the problem. Numerical results demonstrate that the proposed joint design of analog beamforming with AS outperforms conventional array architectures with fixed inter-antenna element spacing.
\end{abstract}

Index Terms - Nonconvex optimization, majorization- minimization, MM, algorithms, analog beamforming, antenna selection

\section{INTRODUCTION}

In the upcoming generation of wireless communication systems (for example, 5G and beyond), the large-scale antenna arrays (LSSAs) become a decisive characteristic for enhancing the performance gains. For example, in the mmWave wireless communication systems, the LSAAs are envisioned to achieve beamforming gains to alleviate the severe propagation losses at mmWave frequencies [1]. For such a system, the fully-digital implementation requires as many radio frequency $(\mathrm{RF})$ chains as the number of antenna elements. Therefore, the power consumption and hardware cost may considerably increase. These drawbacks lead to an interest in analog beamforming or hybrid precoding systems, wherein the beamforming or precoding operation is implemented using an analog architecture [2-7] or a hybrid architecture [8-11], respectively. Herein, our focus is on analog beamforming systems design.

The analog beamforming system design problem has been studied in the literature [2-7]. In general, the analog beamforming is implemented using a phase-shifting architecture, thus, constraining the beamforming vector to be unit-modulus. The optimal beamforming vector is designed by optimizing specific performance criteria subject to the unit-modulus constraints. From a beampattern matching perspective, the problem of analog beamforming design is studied in $[6,7]$.

This work is supported by the National Research Fund (FNR), Luxembourg under the AFR-PPP grant for Ph.D. project SPASAT (Ref.: 11607283), the CORE-PPP project PROSAT, ECLECTIC, CI-PHY and DISBuS.
In addition to the advantages provided by a low-cost analog beamforming architecture employing LSAAs, the problem of antenna selection (AS) becomes important to reduce the power consumption and hardware complexity. Several works study the problem of AS in different applications [12-16]. The problem of designing nonuniformly spaced arrays is considered in [12] where the cost function is chosen to select the array with a minimum number of antenna elements. Some other related works in $[13,15]$ minimize the number of active array elements subject to the constraints on the beampattern. In these applications, the aim is to minimize the total number of active antennas or placing a given number of antennas with beamforming over an array aperture subject to the constraints on the beampattern. This problem is antenna placement (AP) with beamforming. A reconfigurable adaptive array beamforming strategy by AS is studied in [14]. In [16], the problem of transmit AS with analog beamforming is studied by maximizing the spectral efficiency of a multiple-input single-output (MISO) mmWave system. One of the differences between AS and AP is that AS requires a switching network to select a subset of antennas whereas in AP we do not require switches.

The previous works [6,7] do not perform AS to reduce the power consumption and hardware complexity. From an AP perspective, therein, the antenna positions are assumed to be fixed and the corresponding beamforming vector is designed. In comparison to the existing works, herein, we consider the problem of analog beamforming with AS/AP. We approach this problem from a beampattern matching perspective for an analog beamforming system where we provide one of the ways to control the sidelobe levels at the grid points of the sampled beampattern. To this end, we formulate a sparse unit-modulus least-squares (SULS) problem, which is nonconvex and NP-hard due to the unit-modulus [17] and the sparsity constraints. Typically, the unit-modulus constraints are handled using the semidefinite relaxation (SDR) technique which lifts the search space from $M$-dimensions to $M^{2}$-dimensions. This results in a large number of parameters to optimize over, thereby, resulting in more memory storage and computational requirements. Another problem with SDR is that it empirically returns a rank-one solution, but in cases, the solution is not rank-one, an appropriate randomization technique should be employed $[17,18]$. Moreover, the sparsity constraint further increases the difficulty in handling the problem.

To tackle this problem, we propose an efficient and scalable algorithm based on the majorization-minimization (MM) framework [19] to solve the SULS problem. The proposed algorithm converges to a stationary point of the problem. As shown by the numerical results, the proposed approach achieves better sidelobe levels than the conventional antenna arrays with fixed inter-antenna element spacing when LSAAs are employed.

Notation: A vector and a matrix are represented by $\mathbf{a}$ and $\mathbf{A}$ respectively. The $i, j$ element of a matrix is denoted as $\mathbf{A}(i, j)$. The 
$i$-th entry of a vector $\mathbf{a}$ is represented as $\mathbf{a}(i)$ or $a_{i}$. The elementwise operations used on a vector are, $e^{(j \mathbf{a})}$ for complex exponential, $\arg (\mathbf{a})$ for the phase/argument and $|\mathbf{a}|$ for the magnitude. The $\ell_{0^{-}}$ norm of $\mathbf{a}$ and $\mathbf{A}$, counting the number of non-zeros are represented as $\|\mathbf{a}\|_{0}$ and $\|\mathbf{A}\|_{0}$, respectively. Note that for simplicity we refer $\ell_{0}$ as a norm though it is not a norm. A positive semi-definite matrix is denoted as $\mathbf{A} \succeq 0$.

\section{ANALOG BEAMFORMING ARCHITECTURE AND PROBLEM FORMULATION}

In this section, we first present the employed analog beamforming architecture. Then, the problem formulation follows.

\subsection{Analog Beamforming Architecture}

Let us consider a transmitter equipped with an antenna array and each antenna element is connected to a phase-shifting element. For simplicity, we consider a uniform-linear array (ULA) at the transmitter with an inter-element spacing, $d$. For a ULA, the antenna array response in a direction $\theta_{i}$ is given by,

$$
\mathbf{a}\left(\theta_{i}\right)=\left[\begin{array}{lllll}
1 & e^{j \phi_{i}} & e^{j 2 \phi_{i}} & \ldots & e^{j(M-1) \phi_{i}}
\end{array}\right]^{T} .
$$

where $\phi_{i}=2 \pi \frac{d}{\lambda} \sin \theta_{i}$ is an auxiliary variable used to simplify notation. The angle space of interest is discretized into $N$ grid points as $\boldsymbol{\theta}=\left[\theta_{1}, \theta_{2}, \ldots, \theta_{N}\right]^{T}$, where $\theta_{i}$ represents the $i$-th element of vector $\boldsymbol{\theta}$. The antenna array response can be concisely represented in $\boldsymbol{\theta}$ in matrix form as $\mathbf{A}(\boldsymbol{\theta})=\left[\mathbf{a}\left(\theta_{1}\right), \mathbf{a}\left(\theta_{2}\right), \ldots, \mathbf{a}\left(\theta_{N}\right)\right]^{H}$ from all the directions. The beampattern response in $\theta_{i}$ can be written as $y_{i}\left(\theta_{i}\right)=\mathbf{a}\left(\theta_{i}\right)^{H} \mathbf{w}$, where $\mathbf{w}$ is the beamforming vector to be designed with unit-modulus entries. Hereafter for notational simplicity, we will write $y\left(\theta_{i}\right), \mathbf{a}\left(\theta_{i}\right)$ and $\mathbf{A}(\boldsymbol{\theta})$ as $y_{i}, \mathbf{a}_{i}$ and $\mathbf{A}$, respectively. The system of equations relating the beampattern with the beamforming vector can be further written as, $\mathbf{y}=\mathbf{A w}$, where $\mathbf{y}$ is the $N \times 1$ sampled beampattern vector denoting the desired response along the directions represented by vector $\boldsymbol{\theta}$. To perform AS, we introduce a diagonal matrix $\mathbf{Q}$ taking binary values on its diagonal in the system model as,

$$
\mathbf{y}=\mathbf{A Q w} \text {. }
$$

Therefore, the design variables are $\mathbf{w}$ and $\mathbf{Q}$.

\subsection{Problem Formulation}

Now, to address the analog beamforming with AS/AP problem, we formulate the following SULS problem,

$$
\begin{aligned}
\mathcal{P}_{1}: \min _{\mathbf{Q}, \mathbf{w}} & \|\mathbf{y}-\mathbf{A} \mathbf{Q w}\|_{2}^{2} \\
\text { subject to } & \left|w_{i}\right|^{2}=1 \\
& \mathbf{Q}_{i i} \in\{0,1\}, \forall i \in[M] \\
& \mathbf{Q}_{i j}=0, i \neq j, \forall i, j \in[M] \\
& \|\mathbf{Q}\|_{0}=K,
\end{aligned}
$$

where $K$ represents the number of active array elements to be selected out of $M$ available ones. The unit-modulus constraints on w ensure that only the phase of the incoming signal is shifted and the magnitude is kept constant. In comparison to earlier works [6,7], problem $\mathcal{P}_{1}$ has an additional selection variable, $\mathbf{Q}$, to select the subset of the active array elements and the $\ell_{0}$-norm constraint. The unit-modulus least-squares (ULS) problem is shown to be NP-hard and the presence of nonconvex $\ell_{0}$-norm constraint increases further the difficulty.

\section{ALGORITHMIC SOLUTION}

In this section, we develop an efficient and scalable algorithm to solve problem $\mathcal{P}_{1}$. Since the problem is nonconvex and NP-hard, a joint solution in variables $\mathbf{w}$ and $\mathbf{Q}$ is intractable. To solve the problem, one can adopt the alternating minimization framework. In particular, we can minimize the problem with respect to the variables $\mathbf{w}$ and $\mathbf{Q}$ in an alternating fashion. However, the problem with respect to $\mathbf{w}$ given $\mathbf{Q}$, and vice-versa remains NP-hard due to the unit-modulus and combinatorial nature of the constraints, respectively. Generally, the unit-modulus constraints can be handled using the semidefinite relaxation (SDR) technique which lifts the search space from $M$-dimensions to $M^{2}$-dimensions. This results in a large number of parameters to optimize over, thereby, resulting in more memory storage and computational requirements. For the binary variable $\mathbf{Q}$, the problem can be handled by relaxing the binary constraints as the box constraints, $[0,1]$. But the solution obtained using this approach may not lie in the feasible solution set of the original unrelaxed problem, i.e., the binary constraints may not be satisfied. Thus, an appropriate quantization step is necessary to obtain a feasible solution to problem $\mathcal{P}_{1}$. Moreover, in problem $\mathcal{P}_{1}$, the $\ell_{0}$-norm constraint makes the problem further difficult.

To overcome the aforementioned problems with the existing approaches, herein we propose an efficient and scalable algorithm for LSAAs. But before we proceed towards the development of the algorithm, we first cast problem $\mathcal{P}_{1}$ in a more amenable form given by,

$$
\begin{aligned}
\mathcal{P}_{2}: \min _{\mathbf{w}} \quad f(\mathbf{w}) & =\|\mathbf{y}-\mathbf{A} \mathbf{w}\|_{2}^{2} \\
\text { subject to }\left|w_{i}\right|^{2} & \in\{0,1\}, \forall i \in[M] \\
\|\mathbf{w}\|_{0} & =K .
\end{aligned}
$$

In problem $\mathcal{P}_{2}$, we have removed the variable $\mathbf{Q}$ by imposing an additional constraint on the magnitude of the entries of vector $\mathbf{w}$. Now, the magnitude of the entries of vector $\mathbf{w}$ are constrained to be either 0 or 1 instead of being unit-modulus, only. If an antenna element is not active, then, the magnitude of the corresponding element of vector $\mathbf{w}$ should be 0 , otherwise, the magnitude should be 1 to satisfy the unit-modulus constraints. This way, we jointly optimize the beamforming vector and the placement of the active elements of the antenna array without the need to optimize an additional variable, $\mathbf{Q}$. Moreover, in problem $\mathcal{P}_{2}$, the $\ell_{0}$-norm constraint appears on variable w. Thus, to activate $K$ antenna elements the number of non-zero elements in vector $\mathbf{w}$ should be $K$.

\subsection{Algorithm for Problem $\mathcal{P}_{2}$}

Problem $\mathcal{P}_{2}$ is still nonconvex and NP-hard. To develop an efficient solution, we first note that the objective function in $\mathcal{P}_{2}$ is quadratic in variable $\mathbf{w}$. This observation naturally leads to the development of an MM-based algorithm. The basic principle behind the MM framework is to construct a surrogate objective function majorizing the original objective function. To construct a majorizing function, first we expand the objective function $f(\mathbf{w})$ as,

$$
f(\mathbf{w})=\mathbf{y}^{H} \mathbf{y}-2 \operatorname{Re}\left(\mathbf{y}^{H} \mathbf{A} \mathbf{w}\right)+\mathbf{w}^{H} \mathbf{A}^{H} \mathbf{A} \mathbf{w} .
$$

It can be seen that the last term in (3) is a convex quadratic function in $\mathbf{w}$. Therefore, we approximate this term using the quadratic upper bound principle [19]. The following Lemma 3.1 which follows Lemma 1 in [20] generalizes this principle for the specific case of a quadratic function of a vector variable. 
Lemma 3.1 (Lemma 1 in [20]). The quadratic function of the form $\mathbf{a}^{H} \mathbf{P a}$, with $\mathbf{P}$ being a Hermitian matrix is majorized by

$$
\mathbf{a}^{H} \mathbf{T a}+2 \operatorname{Re}\left(\mathbf{a}^{H}(\mathbf{P}-\mathbf{T}) \mathbf{a}_{k}\right)+\mathbf{a}_{k}^{H}(\mathbf{T}-\mathbf{P}) \mathbf{a}_{k}
$$

at the point $\mathbf{a}_{k}$, where $\mathbf{T}$ is a Hermitian matrix such that $\mathbf{T} \succeq \mathbf{P}$.

Lemma 3.1 can be easily proven using the second-order Taylor expansion and subsequently replacing the Hessian matrix by another Hermitian matrix $\mathbf{T}$ such that $\mathbf{T} \succeq \mathbf{P},[19,20]$. Now, using Lemma 3.1 with $\mathbf{T}=\lambda_{\max }(\mathbf{P}) \mathbf{I}$, where $\mathbf{P}=\mathbf{A}^{H} \mathbf{A}$ and $\mathbf{I}$ is a $M \times M$ identity matrix, the last term in (3) can be majorized as,

$$
\begin{aligned}
\mathbf{w}^{H} \mathbf{P} \mathbf{w} \leq & \lambda_{\max }(\mathbf{P}) \mathbf{w}^{H} \mathbf{w} \\
& +2 \operatorname{Re}\left(\mathbf{w}^{H}\left(\mathbf{P}-\lambda_{\max }(\mathbf{P}) \mathbf{I}\right) \mathbf{w}_{k}\right)+\mathbf{w}_{k}^{H} \mathbf{P} \mathbf{w}_{k},
\end{aligned}
$$

where $\mathbf{w}_{k}$ is the solution available at $k$-th iteration. It can be seen that, the first term in (4) is equals to $\lambda_{\max }(\mathbf{P}) K$, which is a constant, and the third term is also independent of $\mathbf{w}$. Thus, after neglecting all the constant terms in (3) and (4), the overall majorized problem to solve at $(k+1)$-th iteration can be written as,

$$
\begin{gathered}
\mathcal{P}_{3}: \min _{\mathbf{w}} \quad-2 \operatorname{Re}\left(\mathbf{w}^{H} \mathbf{d}^{(k)}\right) \\
\text { subject to }\left|w_{i}\right|^{2} \in\{0,1\}, \forall i \in[M] \\
\|\mathbf{w}\|_{0}=K,
\end{gathered}
$$

where $\mathbf{d}^{(k)}=\left(\mathbf{A}^{H} \mathbf{y}-(\mathbf{P}-\beta \mathbf{I}) \mathbf{w}^{(k)}\right)$ and $\beta=\lambda_{\max }(\mathbf{P})$. Problem $\mathcal{P}_{3}$ can be equivalently written as,

$$
\begin{array}{cl}
\mathcal{P}_{4}: \max _{\mathbf{w}} & \operatorname{Re}\left(\mathbf{w}^{H} \mathbf{d}^{(k)}\right) \\
\text { subject to } & \left|w_{i}\right|^{2} \in\{0,1\}, \forall i \in[M] \\
\|\mathbf{w}\|_{0}=K .
\end{array}
$$

Remark 1. Benchmark Solution: Note that for the comparison between conventional and the proposed design, we also solve problem $\mathcal{P}_{4}$ with only unit-modulus constraints. It can be shown that without introducing 0 in the feasible set of $\mathbf{w}$ and $\ell_{0}$-norm constraint, the problem can be iteratively solved using the aforementioned MM framework in closed-form like the special case of problem $\mathcal{P}_{1}$ in [7].

To solve problem $\mathcal{P}_{4}$, first, we observe that the selection of an antenna is indicated by the magnitude of the corresponding entry, $w_{i}$, and it is not affected by its phase. Moreover, there are no constraints on the phase of $w_{i}$. Thus, we exploit this observation and first write the optimal solution of $\arg (\mathbf{w})$ as,

$$
\arg (\mathbf{w})=\arg \left(\mathbf{d}^{(k)}\right)+2 k \pi, \forall k=0, \pm 1, \pm 2, \ldots
$$

Substituting the above solution into the objective function in $\mathcal{P}_{4}$, we arrive at the following problem,

$$
\begin{gathered}
\mathcal{P}_{5}: \max _{|\mathbf{w}|}|\mathbf{w}|^{T}\left|\mathbf{d}^{(k)}\right| \\
\text { subject to }\left|w_{i}\right|^{2} \in\{0,1\}, \forall i \in[M] \\
\|\mathbf{w}\|_{0}=K .
\end{gathered}
$$

It can be seen that the objective function in problem $\mathcal{P}_{5}$ is maximized when $\left|w_{i}\right|=1$ for all $i$ in $[M]$. But the $\ell_{0}$-norm constraint enforces only $K$ non-zero entries. Thus, the optimal solution to problem $\mathcal{P}_{5}$ can be obtained by sorting the vector $\left|\mathbf{d}^{(k)}\right|$ as given by,

$$
\left|d_{i_{1}}^{(k)}\right| \geq\left|d_{i_{2}}^{(k)}\right| \geq \cdots \geq\left|d_{i_{M}}^{(k)}\right| .
$$

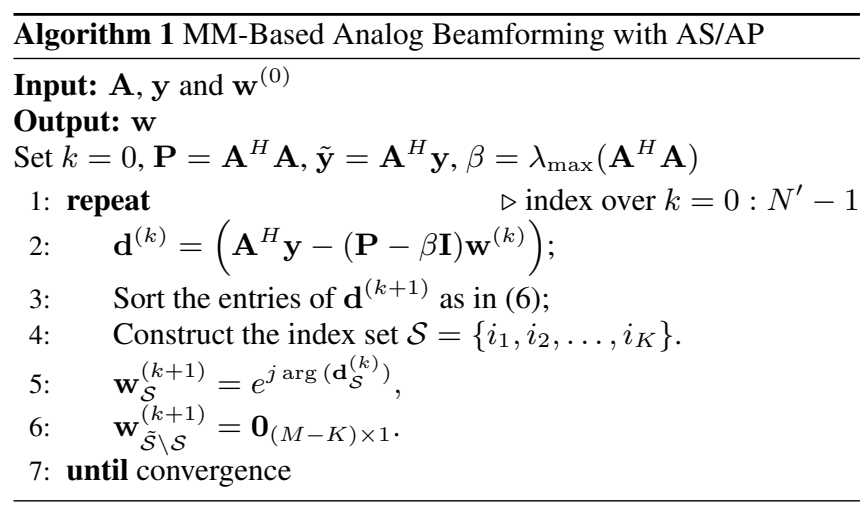

Now, we pick the first $K$ largest elements of $|\mathbf{w}|$ and setting the corresponding entries in vector $|\mathbf{w}|$ to 1 and the remaining entries to 0 . Let $\tilde{\mathcal{S}}=\left\{i_{1}, i_{2}, \ldots, i_{M}\right\}$ and $\mathcal{S}=\left\{i_{1}, i_{2}, \ldots, i_{K}\right\}$ denote the index sets corresponding to the sorted (in descending order) and first $K$ largest entries of vector $\mathbf{d}^{(k)}$, respectively. Therefore, after combining (5) and the entries selected using the index set $\mathcal{S}$, we obtain the the optimal solution to problem $\mathcal{P}_{4}$ as given by,

$$
\mathbf{w}_{\mathcal{S}}=e^{j \arg \left(\mathbf{d}_{\mathcal{S}}^{(k)}\right)}
$$

where $\mathbf{d}_{\mathcal{S}}^{(k)}$ and $\mathbf{w}_{\mathcal{S}}$ denote the $K$ dimensional vector with entries selected from the index set $\mathcal{S}$. The rest of the $M-K$ entries are equal to zero. The procedure is presented in Algorithm 1. Note that all the entries of vector $\mathbf{w}^{(0)}$ in Algorithm 1 are initialized to be unit-modulus. The worst-case per-iteration computational complexity of Algorithm 1 is $O\left(N^{2}\right)$. The convergence of Algorithm 1 is established via the following theorem.

Theorem 3.2. Let $\left\{\mathbf{w}^{(k)}\right\}$ be a sequence generated by Algorithm 1. This solution sequence converges to a stationary point of problem $\mathcal{P}_{2}$.

Proof. Since the underlying constraint set is compact, the convergence of the iterates obtained from Algorithm 1 to a stationary point can be established by working on similar lines to the proof of Theorem 5 in [20].

\section{SIMULATION RESULTS}

In this section, indicative numerical results are shown to evaluate the performance of the proposed approach. We consider the objective function of the problem, i.e., the least-squares approximation of the desired beampattern as a performance metric to compare the results with conventional beamforming design. Kindly refer to Remark 1 for benchmark design based on the conventional analog beamforming with fixed inter-antenna element spacing. The beampattern vector $\mathbf{y}$ is generated according to (8) below,

$$
y_{i}= \begin{cases}1 & \text { if } i \in \mathcal{I} \\ 0 & \text { otherwise }\end{cases}
$$

where $\mathcal{I} \in[1, N]$ denotes the index set for non-zero entries of vector y. We consider two scenarios, namely, when the two arrays have the same and different apertures. 


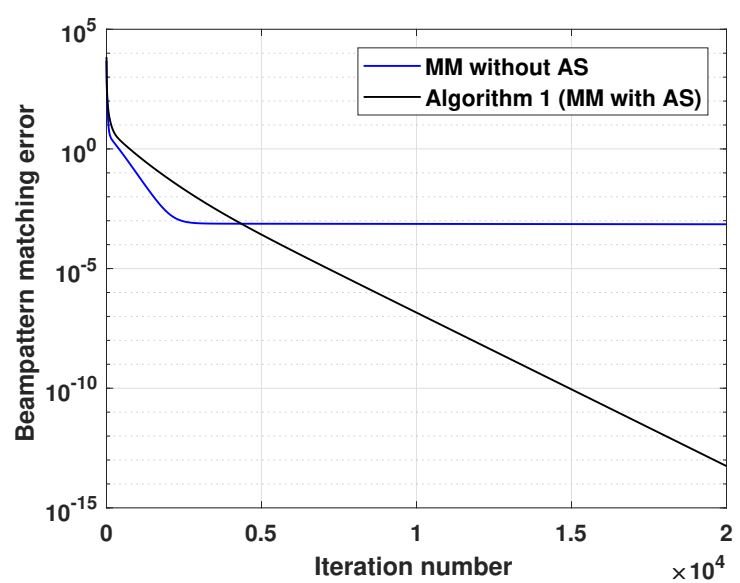

(a) Objective function evolution.

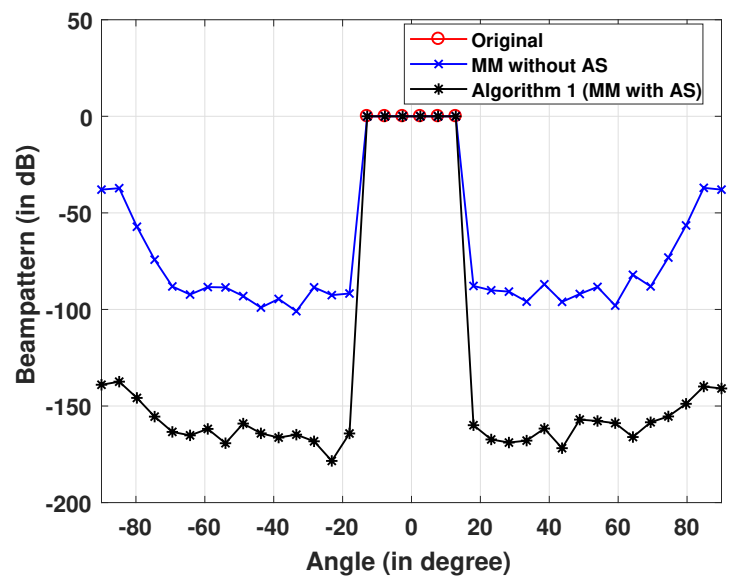

(b) Sampled beampatterns obtained from different algorithms (refer to (8)).

Fig. 1: Same array apertures, $M=219, K=110$ and $N=36$.

\subsection{Same Aperture to that of Conventional Array}

The angle space from $\left[-90^{\circ}, 90^{\circ}\right]$ is discretized into $N=36$ points with set $\mathcal{I}$ corresponding to the angle range $\left[-15^{\circ}, 15^{\circ}\right]$. We consider two arrays with the same antenna array aperture with spacing $d_{1}=\frac{\lambda}{2}$ and $d_{2}=\frac{\lambda}{4}$. We consider a ULA with $K=110$ antenna elements with $d_{1}$ spacing. The array with $d_{2}$ spacing is having $M=219$ number of antenna elements $(M>K)$ but only $K=110$ of them are active. The beampattern matching error comparison without AS is shown in Fig. 1a and the corresponding sampled beampatterns obtained are shown in Fig. 1b. It can be seen that the proposed approach for analog beamforming design with AS/AP performs better and achieves a lower sidelobe level in comparison to the one without AS. This is due to the fact that the array with more grid positions has more degrees of freedom to select/place the antennas. Thus, more efficient beamforming performance.

\subsection{Different Aperture to that of Conventional Array}

The angle space $\left[-90^{\circ}, 90^{\circ}\right]$ is discretized using $N=60$ points with the set $\mathcal{I}$ corresponding to the angle range $\left[-15^{\circ}, 15^{\circ}\right]$. In this case, we consider $d_{1}=d_{2}=\frac{\lambda}{2}, M=500$ and $K=240$. The antenna array without AS is a ULA formed with uniform spacing $d_{1}$ spacing and $K=240$ antennas. For analog beamforming with $\mathrm{AS}, K=$

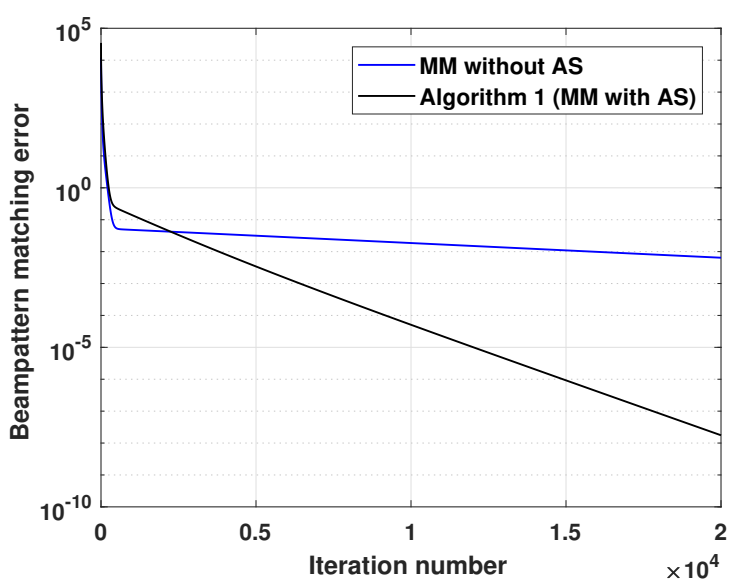

(a) Objective function evolution.

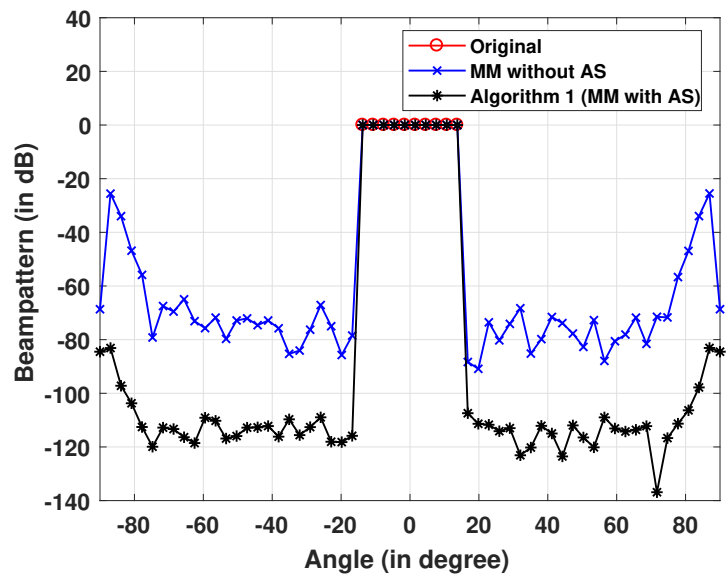

(b) Sampled beampatterns obtained from different algorithms (refer to (8)).

Fig. 2: Different array apertures, $M=500, K=240$ and $N=60$.

240 antennas are selected out of $M=500$ antennas. In this case, both the arrays have a different aperture. The beampattern matching error variation is shown in Fig. 2a and the corresponding sampled beampatterns obtained using different algorithms are shown in Fig. $2 \mathrm{~b}$. Once again, the analog beamforming with AS/AP achieves better beampattern matching accuracy and the lower sidelobe level because of more degrees of freedom to select/place the antennas and larger antenna aperture.

\section{CONCLUSION}

In this work, we proposed a novel approach for designing the ana$\log$ beamforming with antenna selection (AS) or antenna placement (AP) for the wireless communication systems employing large-scale antenna arrays (LSAAs). We formulated a beampattern matching problem for the joint design as a sparse unit-modulus least-squares (SULS) problem. We developed an efficient and scalable algorithm to solve the problem with a theoretical convergence guarantee based on the majorization-minimization (MM) framework. Numerical results demonstrated that the proposed algorithm by jointly designing analog beamforming with AS/AP outperforms the conventional array architectures with fixed inter-antenna element spacing when LSAAs are employed. 


\section{REFERENCES}

[1] A. Alkhateeb, O. El Ayach, G. Leus, and R. W. Heath, "Channel estimation and hybrid precoding for millimeter wave cellular systems," IEEE J. Sel. Topics Signal Process., vol. 8, no. 5, pp. 831-846, Oct 2014.

[2] S. T. Smith, "Optimum phase-only adaptive nulling," IEEE Trans. Signal Process., vol. 47, no. 7, pp. 1835-1843, July 1999.

[3] P. Thompson, "Adaptation by direct phase-shift adjustment in narrow-band adaptive antenna systems," IEEE Trans. Antennas Propag., vol. 24, no. 5, pp. 756-760, Sep. 1976.

[4] C. Baird and G. Rassweiler, "Adaptive sidelobe nulling using digitally controlled phase-shifters," IEEE Trans. Antennas Propag., vol. 24, no. 5, pp. 638-649, Sep. 1976.

[5] T. H. Ismail and M. M. Dawoud, "Null steering in phased arrays by controlling the element positions," IEEE Trans. Antennas Propag., vol. 39, no. 11, pp. 1561-1566, Nov 1991.

[6] J. Tranter, N. D. Sidiropoulos, X. Fu, and A. Swami, "Fast unit-modulus least squares with applications in beamforming," IEEE Trans. Signal Process., vol. 65, no. 11, pp. 2875-2887, June 2017.

[7] A. Arora, C. G. Tsinos, B. Shankar Mysore R, S. Chatzinotas, and B. Ottersten, "Majorization-minimization algorithms for analog beamforming with large-scale antenna arrays," in 2019 IEEE Global Conference on Signal and Information Processing (GlobalSIP), 2019, pp. 1-5.

[8] A. Arora, C. G. Tsinos, B. S. M. R. Rao, S. Chatzinotas, and B. Ottersten, "Hybrid transceivers design for large-scale antenna arrays using majorization-minimization algorithms," IEEE Trans. Signal Process., vol. 68, pp. 701-714, 2020.

[9] C. G. Tsinos, S. Maleki, S. Chatzinotas, and B. Ottersten, "On the energy-efficiency of hybrid analog-digital transceivers for single- and multi-carrier large antenna array systems," IEEE J. Sel. Areas Commun., vol. 35, no. 9, pp. 1980-1995, 2017.

[10] C. G. Tsinos, S. Chatzinotas, and B. Ottersten, "Hybrid analogdigital transceiver designs for multi-user MIMO mmWave cognitive radio systems," IEEE Trans. Cogn. Commun. Netw., vol. 6, no. 1, pp. 310-324, 2020.

[11] A. Arora, C. G. Tsinos, B. S. Mysore R., S. Chatzinotas, and B. Ottersten, "MM-based solution for partially connected hybrid transceivers with large scale antenna arrays," in 2019 IEEE Global Communications Conference (GLOBECOM), 2019, pp. 1-6.

[12] R. M. Leahy and B. D. Jeffs, "On the design of maximally sparse beamforming arrays," IEEE Trans. Antennas Propag., vol. 39, no. 8, pp. 1178-1187, 1991.

[13] S. E. Nai, W. Ser, Z. L. Yu, and H. Chen, "Beampattern synthesis for linear and planar arrays with antenna selection by convex optimization," IEEE Trans. Antennas Propag., vol. 58, no. 12, pp. 3923-3930, 2010.

[14] X. Wang, E. Aboutanios, M. Trinkle, and M. G. Amin, "Reconfigurable adaptive array beamforming by antenna selection," IEEE Trans. Signal Process., vol. 62, no. 9, pp. 2385-2396, 2014.

[15] R. C. Nongpiur and D. J. Shpak, "Synthesis of linear and planar arrays with minimum element selection," IEEE Trans. Signal Process., vol. 62, no. 20, pp. 5398-5410, 2014.
[16] H. Li, Q. Liu, Z. Wang, and M. Li, "Transmit antenna selection and analog beamforming with low-resolution phase shifters in mmwave MISO systems," IEEE Commun. Lett., vol. 22, no. 9, pp. 1878-1881, 2018.

[17] Shuzhong. Zhang and Yongwei. Huang, "Complex quadratic optimization and semidefinite programming," SIAM J. Optim., vol. 16 , no. 3, pp. 871-890, 2006.

[18] Z. Luo, W. Ma, A. M. So, Y. Ye, and S. Zhang, "Semidefinite relaxation of quadratic optimization problems," IEEE Signal Process. Mag., vol. 27, no. 3, pp. 20-34, May 2010.

[19] David R Hunter and Kenneth Lange, "A tutorial on MM algorithms," Am. Statist., vol. 58, no. 1, pp. 30-37, 2004.

[20] J. Song, P. Babu, and D. P. Palomar, "Optimization methods for designing sequences with low autocorrelation sidelobes," IEEE Trans. Signal Process., vol. 63, no. 15, pp. 3998-4009, Aug 2015. 